\title{
A Characterization of a Class of Anomalous Solids
}

\author{
Kokichi SUGIHARA \\ Department of Mathematical Informatics, Graduate School of Information Science and Technology, \\ University of Tokyo, 7-3-1 Hongo, Bunkyo-ku, Tokyo 113-8656, Japan \\ E-mail:sugihara@mist.i.u-tokyo.ac.jp
}

Received April 1, 2005; final version accepted May 4, 2005

\begin{abstract}
Objects represented by anomalous pictures are usually considered unrealizable in a three-dimensional space, but some of them are actually realizable. This paper characterizes a class of realizable anomalous pictures from a mathematical point of view. Distribution of degrees of freedom in the choice of depths of the vertices of a polyhedron represented by a picture is studied, and a decomposition of a polyhedron into components with the minimum degrees of freedom is proposed. According to this decomposition, a class of realizable anomalous pictures is characterized.
\end{abstract}

KEYWORDS: Anomalous picture, freedom in depth, polyhedron, tight component

\section{Introduction}

"Anomalous pictures", also called "pictures of impossible objects", are familiar and attract many people because they generate interesting optical illusion to human eyes. Actually, when we see these pictures, we have some impression of three-dimensional object structures, but at the same time we feel that those objects cannot be realized in the three-dimensional space. Anomalous pictures are also famous in that they are often used by artists such as Escher (1993) (see also Ernst (1992)) and Anno (1974).

These pictures were studied from a scientific point of view in visual psychology (Gregory, 1971; Penrose and Penrose, 1958; Robinson, 1972) in geometry (Cowan, 1974, 1977; Sugihara, 1982; Térouanne, 1983), and in artificial intelligence (Clowes, 1971; Draper, 1978, 1981; Huffman, 1971).

Object structures represented by anomalous pictures are usually considered unrealizable. People usually think that anomalous pictures are just pictures in the two-dimensional world, and the solids represented by those pictures cannot exist in the three-dimensional space; they exist only in human imagination.

Contrary to this common sense, however, some of such objects can actually be realized in the three-dimensional space if we use some tricky techniques. A famous technique is to generate a gap in the depth of the object from the viewer. We can generate a solid in such a way that two mutually disconnected parts look connected when seen from a special view point, and thus its projection coincides with an anomalous picture. This technique is also used in artists such as Fukuda (Ernst, 1992; Sugihara and Hayakawa, 1997).

Another technique is to use curved surfaces although they look planar (Ernst, 1992). Any objects represented by anomalous pictures can be realized in the three-dimensional space if we are allowed to use curved surfaces. However, the resulting objects are usually difficult to cheat human eyes because it is almost impossible to tune the illumination condition so that curved surfaces look planar.

Still another technique, proposed by the author, is to use the degrees of freedom in the choice of objects represented by the pictures (Sugihara, 1984, 1997, 2004). If a picture represents a solid object, there are infinitely many different objects whose projections coincide with the same picture. So we can generate objects from some of anomalous pictures by choosing one of possible objects carefully.

In this context, it is natural to ask which anomalous pictures are realizable by this method. This paper tries to answer this question. We concentrate our attention on the distribution of degrees of freedom in the choice of objects from a picture, and propose a decomposition of the object structure into maximal substructures with minimum degrees of freedom. Using this decomposition we characterize a class of anomalous pictures that can be realized in the threedimensional space without tricky depth gaps or curved surfaces.

\section{Polyhedral Objects and Their Incidence Structures}

We consider a polyhedral object, i.e., a solid object in the three-dimensional space bounded only by planar faces. The boundary of a polyhedral object consists of a finite number of planar polygons possibly with polygonal holes; we call those planar polygons faces of the polyhedral object. A line segment shared by the boundaries of two faces is called an edge, and a point incident to three or more faces is called a vertex.

Let $P$ be a polyhedral object fixed in the three-dimensional space with $(x, y, z)$ coordinate system. We assume that no face is parallel to the $z$ axis. We denote the set of faces of $P$ by $F(P)$, and the set of vertices of $P$ by $V(P)$. For each 
vertex $v_{i} \in V(P)$, let $\left(x_{i}, y_{i}, z_{i}\right)$ be its coordinates. For each face $f_{j} \in F(P)$, let

$$
a_{j} x+b_{j} y+z+c_{j}=0
$$

be the equation of the plane that contains $f_{j}$. Note that the coefficient of $z$ is fixed to be 1 , and consequently we cannot represent planes that are parallel to the $z$ axis. However, there is no problem because we assumed that no face is parallel to the $z$ axis. We call $a_{j}, b_{j}, c_{j}$ the face parameters of the face $f_{j}$.

Let $I(P)$ be the set of vertex-face pairs $\left(v_{i}, f_{j}\right)$ such that the vertex $v_{i}$ is on the face $f_{j}$. We call $I(P)$ the incidence set of $P$, and call its elements incidence pairs.

Suppose that $\left(v_{i}, f_{j}\right) \in I(P)$. Then, the vertex $v_{i}$ is on the face $f_{j}$, and hence the coordinates $\left(x_{i}, y_{i}, z_{i}\right)$ of $v_{i}$ satisfy the Eq. (1). Hence we get

$$
a_{j} x_{i}+b_{j} y_{i}+z_{i}+c_{j}=0 \text {. }
$$

\section{Reconstruction of Polyhedral Objects from Pictures}

Suppose that, as shown in Fig. 1, the polyhedral object $P$ is projected onto the $x y$ plane orthographically. Then, we get the projected picture, which we denote by $D(P)$.

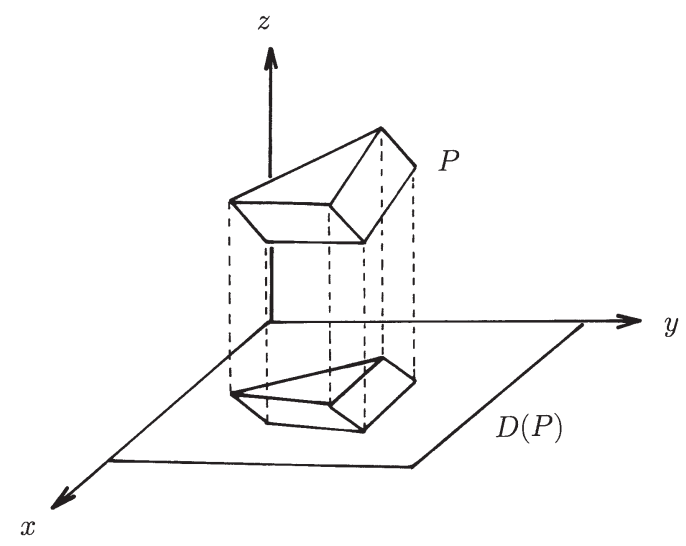

Fig. 1. Polyhedral object and its projection.

There are two typical ways in drawing the projected picture. One is to draw only visible edges, and the other is to draw both visible and invisible edges, visible edges by solid lines and invisible edges by broken lines. In this paper, we consider both types of drawings. When the projected picture represents only visible edges, we consider $P$ as the structure of the visible part of the polyhedral object. When the picture represents both visible and invisible edges, on the other hand, we consider $P$ as the whole structure of the polyhedral object.

For vertex $v_{i} \in V(P)$ and face $f_{j} \in F(P)$, let $v_{i}^{\prime}$ and $f_{j}^{\prime}$ denote the associated projected point and the associated projected face respectively. Since $v_{i}=\left(x_{i}, y_{i}, z_{i}\right)$, we get $v_{i}^{\prime}=\left(x_{i}, y_{i}, 0\right)$. We call $z_{i}$ the height of the vertex. The projected face $f_{j}^{\prime}$ is actually a polygon possibly with polygonal holes; it does not degenerate to a line segment because we assumed that the plane containing the face is not parallel to the $z$ axis.

Now suppose that we are given the incidence set and the projected picture, but that the original polyhedral object $P$ itself is not known. Then, for each vertex $v_{i}$, we know $x_{i}$ and $y_{i}$, but $z_{i}$ is an unknown. Also for each face $f_{j}$, the associated face parameters $a_{j}, b_{j}$ and $c_{j}$ are all unknowns. Therefore, the Eq. (2) is linear in the unknowns $z_{i}, a_{j}, b_{j}$ and $c_{j}$. Recall that the Eq. (2) is obtained for each incidence pair. Gathering such equations for all incidence pairs in $I(P)$, we get a system of linear equations, which we denote by

$$
A \omega=0,
$$

where $\omega$ is the unknown vector $\omega=\left(z_{1}, \ldots, z_{n}, a_{1}, b_{1}, c_{1}, \ldots, a_{m}, b_{m}, c_{m}\right)^{\mathrm{t}}, n=|V(P)|, m=|F(P)|$, and $A$ is an $|I(P)| \times$ $(n+3 m)$ constant matrix.

The vertex heights $z_{1}, z_{2}, \ldots, z_{n}$ and the face parameters $a_{1}, b_{1}, c_{1}, \ldots, a_{m}, b_{m}, c_{m}$ of the original polyhedral object $P$ satisfy (3). However, the solution of (3) is not unique in general. If there is another polyhedral object $Q$ with the same incidence set and the same projected picture, i.e., $I(P)=I(Q)$ and $D(P)=D(Q)$, then the vertex heights and the face parameters associated with $Q$ also satisfies (3). In this sense, the set of solutions of (3) corresponds to the set of polyhedral objects with the same projected picture $D(P)$. 


\section{Degrees of Freedom in the Choice of Objects}

Let us define $\sigma(P)$ by

$$
\sigma(P)=n+3 m-\operatorname{rank}(A) .
$$

Note that $n+3 m$ is the number of unknowns and $\operatorname{rank}(A)$ is the number of linearly independent equations in (3). Hence, $\sigma(P)$ represents the degrees of freedom in the choice of polyhedral objects that have the same projected picture as $P$. We will consider how the degrees of freedom can be used in designing anomalous solid objects.

In order to understand the minimum degrees of freedom, we consider the following transformation in the threedimensional space:

$$
\left(\begin{array}{l}
x^{\prime} \\
y^{\prime} \\
z^{\prime}
\end{array}\right)=\left(\begin{array}{lll}
1 & 0 & 0 \\
0 & 1 & 0 \\
\alpha & \beta & \gamma
\end{array}\right)\left(\begin{array}{l}
x \\
y \\
z
\end{array}\right)+\left(\begin{array}{l}
0 \\
0 \\
\delta
\end{array}\right), \quad \gamma \neq 0 .
$$

Suppose that the point $(x, y, z)$ moves in the polyhedral object $P$, and let $Q$ be the set of points $\left(x^{\prime}, y^{\prime}, z^{\prime}\right)$ obtained by the transformation (4). Since this is a linear transformation, a line is transformed to a line and a plane is transformed to a plane. Therefore, $Q$ is also a polyhedral object.

Thus, if a picture represents a polyhedral object, any objects obtained by this transformation also have the same projected picture as the original object. The transformation (4) contains four parameters $\alpha, \beta, \gamma$ and $\delta$, and consequently, for any picture that represents a polyhedral object correctly, there are at least four degrees of freedom in the choice of the polyhedral object.

This fact can be understood intuitively in the following way. For a given picture, let us choose any one face. Giving the heights of three vertices on that face arbitrarily, we can fix the face in the three-dimensional space. Next let us choose another face that shares an edge with the first face, and give the height of a vertex that is on the second face but not on the first face in such a way that the first and the second face are not coplanar. By this, we can fix the second face. This process is always possible, and hence there are at least four degrees of freedom in fixing a polyhedral object in the three-dimensional space.

If $\gamma<0$, the transformation (4) changes the orientation of the object, that is, the front part and the rear part are reversed. In that case the visible part changes, and hence the corresponding picture also changes in an ordinary sense of a picture. Therefore, in what follows we assume $\gamma>0$. Still we have at least four degrees of freedom in the choice of the object.

Let $X$ be any subset of the face set $F(P)$. We denote by $V(X)$ the set of vertices on a face in $X$, and by $I(X)$ the set of incidence pairs in $I(P)$ whose first element is in $V(X)$ and the second element is in $X$. In other words, we define

$$
\begin{aligned}
I(X) & =I(P) \cap(V \times X), \\
V(X) & =\{v \in V \mid \text { there exists face } f \in X \text { such that }(v, f) \in I(P)\} .
\end{aligned}
$$

We can consider the collection of faces in $X$ together with the associated vertices and incidence pairs. We call this collection the substructure associated with $X$.

Furthermore, let $A(X)$ be the submatrix of $A$ consisting of the rows corresponding to incidence pairs in $I(X)$ and the columns corresponding to the heights of vertices in $V(X)$ and the parameters of faces in $X$. We define $\sigma(X)$ by

$$
\sigma(X)=|V(X)|+3|X|-\operatorname{rank}(A(X)) .
$$

The integer $\sigma(X)$ represents the degrees of freedom in the choice of the substructure associated with $X$.

The next property is not difficult to derive, but since we want to refer to this property later, let us state it in the form of a proposition.

Proposition 1. Let $X_{1}$ and $X_{2}$ be subsets of $F(P)$ such that $X_{1} \cup X_{2}=F(P)$.

(1) If $X_{1} \cap X_{2}=\emptyset$ and $\left|V\left(X_{1}\right) \cap V\left(X_{2}\right)\right| \leq 2$, then

$$
\sigma\left(X_{1} \cup X_{2}\right)=\sigma\left(X_{1}\right)+\sigma\left(X_{2}\right)-\left|V\left(X_{1}\right) \cap V\left(X_{2}\right)\right| .
$$

(2) If $\left|X_{1} \cap X_{2}\right|=1$ and $V\left(X_{1}\right) \cap V\left(X_{2}\right)=F\left(X_{1} \cap X_{2}\right)$,

$$
\sigma\left(X_{1} \cup X_{2}\right)=\sigma\left(X_{1}\right)+\sigma\left(X_{2}\right)-3 .
$$

Proof. First, assume that $X_{1} \cap X_{2}=\emptyset$ and $\left|V\left(X_{1}\right) \cap V\left(X_{2}\right)\right| \leq 2$. Suppose that we fix the substructure consisting of the faces in $X_{1}$ in the three-dimensional space by choosing the heights of $\sigma\left(X_{1}\right)$ vertices. This implies that the heights of the vertices in $V\left(X_{1}\right) \cap V\left(X_{2}\right)$ are fixed also in the space. Hence there remain $\sigma\left(X_{2}\right)-\left|V\left(X_{1}\right) \cap V\left(X_{2}\right)\right|$ degrees of freedom in the substructure consisting of the faces in $X_{2}$. Therefore in total we get 


$$
\sigma\left(X_{1} \cup X_{2}\right)=\sigma\left(X_{1}\right)+\sigma\left(X_{2}\right)-\left|V\left(X_{1}\right) \cap V\left(X_{2}\right)\right| .
$$

Next assume that $\left|X_{1} \cap X_{2}\right|=1$ and $V\left(X_{1}\right) \cap V\left(X_{2}\right)=F\left(X_{1} \cap X_{2}\right)$. This means that the associated two substructures share exactly one common face and the vertices on it. Therefore, fixing the substructure consisting of the faces in $X_{1}$ implies that the common face together with the vertices on it are also fixed, which implies that three degrees of freedom in the substructure associated with $X_{2}$ are removed. Hence, we get

$$
\sigma\left(X_{1} \cup X_{2}\right)=\sigma\left(X_{1}\right)+\sigma\left(X_{2}\right)-3
$$

This proposition helps us to compute the degrees of freedom of a polyhedral object $P$ in a step-by-step manner. Next, let us consider basic components to compute the total degrees of freedom.

\section{Decomposition of a Polyhedron According to the Distribution of Degrees of Freedom}

As we have seen, any correct picture has at least four degrees of freedom in the choice of the associated polyhedral object. In order to study the distribution of degrees of freedom, therefore, substructures of the picture with exactly four degrees of freedom play an important role. Keeping this in mind, we introduce the next definitions.

Subset $X \subseteq F(P)$ is called a tight component if $\sigma(X)=4$ and $\sigma(X \cup Y) \geq 5$ for any nonempty set $Y \subseteq F(P)-X$. That is, a tight component is a maximal substructure with four degrees of freedom. A polyhedral object $P$ is called tight if $X=F(P)$ is the only tight component of $P$, and is called loose if $P$ has two or more tight components.

Let $X$ be a set consisting of two faces that share an edge, as shown in Fig. 2. Then, the degrees of freedom of $X$ is exactly four, because we have three degrees of freedom to fix one of the faces to the three-dimensional space, and one more degree of freedom to fix the other face. We are not interested in this kind of a component. So we call tight component $X$ trivial if $X$ consists of two faces sharing an edge, and nontrivial otherwise.

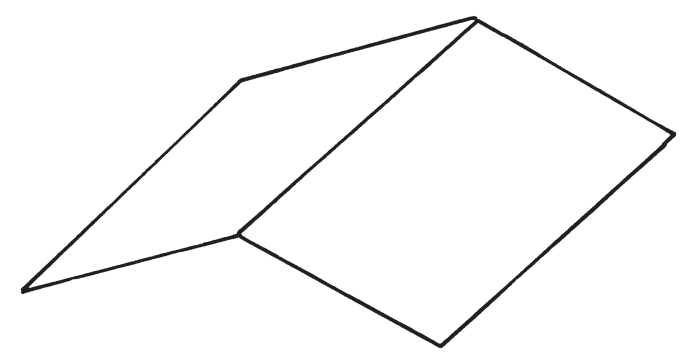

Fig. 2. Two faces sharing an edge.

For a given polyhedral object $P$, let $\left\{X_{1}, X_{2}, \ldots, X_{k}\right\}$ be the set of all nontrivial tight components of $P$, and let us define $Y=F(P)-X_{1} \cup X_{2} \cup \cdots \cup X_{k}$. We call the set $\left\{X_{1}, X_{2}, \ldots, X_{k}, Y\right\}$ the tight-component decomposition of $P$, and call $Y$ the residual part of the tight-component decomposition. A typical example of a residual part is a connected part of a surface consisting of only triangular faces. If the faces are triangular, the Eq. (2) gives no essential constraint on the heights of the vertices; the degree of freedom coincides with the number of vertices. Hence, this part conveys no interesting structure in the distribution of the degrees of freedoms. If $Y=\emptyset$, we abbreviate the tight-component decomposition $\left\{X_{1}, X_{2}, \ldots, X_{k}, Y\right\}$ by $\left\{X_{1}, X_{2}, \ldots, X_{k}\right\}$.

For example, let us consider the polyhedral objects represented by the pictures in Fig. 3, where the visible parts of the objects are represented by solid lines whereas the invisible parts are represented by broken lines. The object in (a) is tight, while the object in (b) is loose. Actually the object in (b) consists of two tight components, i.e., the left wall and the right wall. Actually, the object in Fig. 3(b) has six degrees of freedom, because the two tight components have two common vertices but have no common face, which is the case (1) in Proposition 1.

Next consider another example of an object shown in Fig. 4, where four rectangular columns are standing on the top face of a base brick. This object has five tight components, the base brick and the four columns, and all the five tight components have the top face of the base brick in common. The picture of this object has eight degrees of freedom in fixing the shape in the space. This can be understood in the following way.

Let $X_{1}, X_{2}, \ldots, X_{5}$ denote the five tight components of this object, $X_{1}$ representing the base brick and $X_{2}, X_{3}, X_{4}$ and $X_{5}$ representing the four rectangular columns. First, consider $X_{1}$ and $X_{2}$. Since they share a common face, we get from Proposition 1

$$
\sigma\left(X_{1} \cup X_{2}\right)=\sigma\left(X_{1}\right)+\sigma\left(X_{2}\right)-3=4+4-3=5 .
$$

Next, consider the two substructures $X_{1} \cup X_{2}$ and $X_{3}$. Since they also share a common face, we apply Proposition 1 again and obtain 


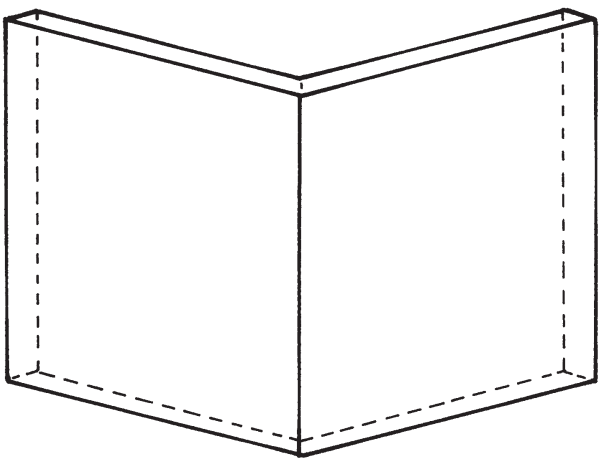

(a)

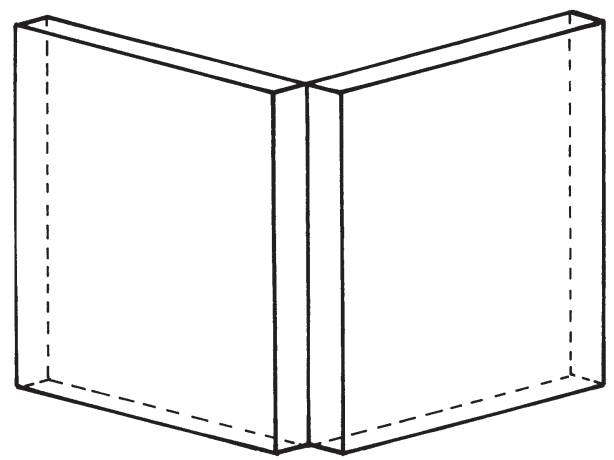

(b)

Fig. 3. Objects: (a) tight; (b) loose.

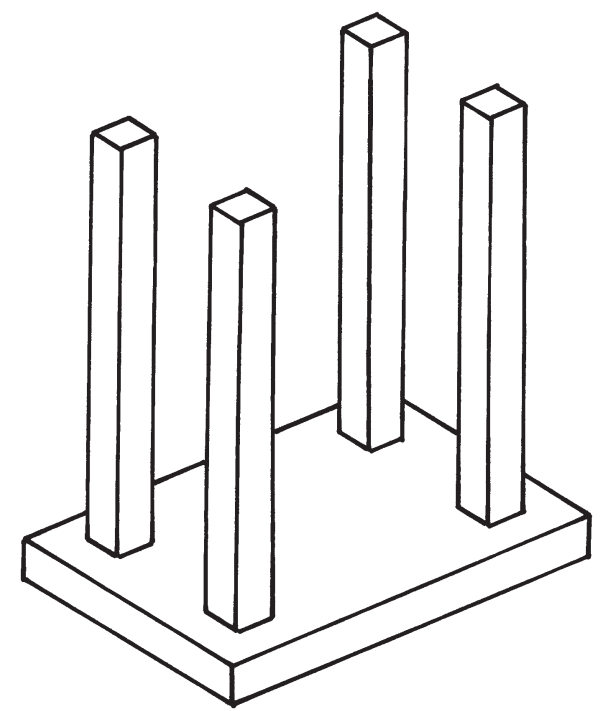

Fig. 4. Object with four columns.

$$
\sigma\left(X_{1} \cup X_{2} \cup X_{3}\right)=\sigma\left(X_{1} \cup X_{2}\right)+\sigma\left(X_{3}\right)-3=5+4-3=6 .
$$

Repeating the same procedure two more times we get

$$
\begin{aligned}
\sigma\left(X_{1} \cup X_{2} \cup X_{3} \cup X_{4} \cup X_{5}\right) \\
\quad=\sigma\left(X_{1} \cup X_{2} \cup X_{3} \cup X_{4}\right)+\sigma\left(X_{5}\right)-3 \\
\quad=\left(\sigma\left(X_{1} \cup X_{2} \cup X_{3}\right)+\sigma\left(X_{4}\right)-3\right)+\sigma\left(X_{5}\right)-3 \\
\quad=8 .
\end{aligned}
$$

\section{Design of Anomalous Solids}

Both of the objects in Fig. 3 consist of two walls. Making a window through each wall, we get the objects shown in Fig. 5. In this picture the visible parts only are drawn for simplicity, but let us assume that invisible parts are also drawn and consider $P$ as the whole structure consisting of both the visible part and the invisible part.

Making this kind of windows does not change the degrees of freedom of the whole objects, and hence the object in Fig. 5(a) is tight while that in Fig. 5(b) is loose.

A typical method for generating an anomalous picture is to insert an additional object in such a way that visible part and hidden part are locally exchanged. Examples are shown in Fig. 6, where a rod is inserted through the windows of the objects in an unusual way.

Now, we are interested in asking whether or not the objects drawn in Fig. 6(a) and (b) are realizable as the threedimensional solid objects. To make our question clearer, we place the following assumption.

Assumption 1. The additional rod is connected and straight. 
1

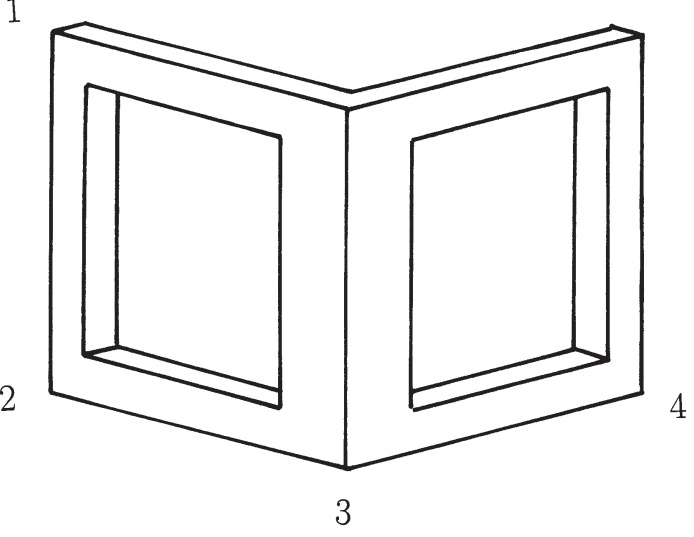

(a)
3

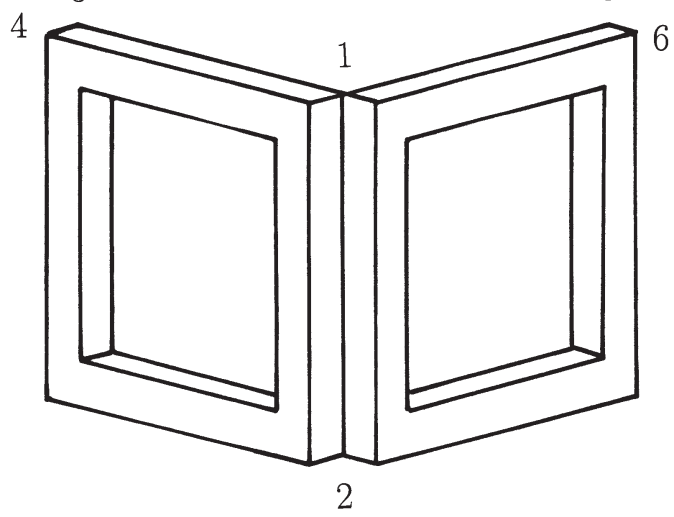

(b)

Fig. 5. Objects with windows.

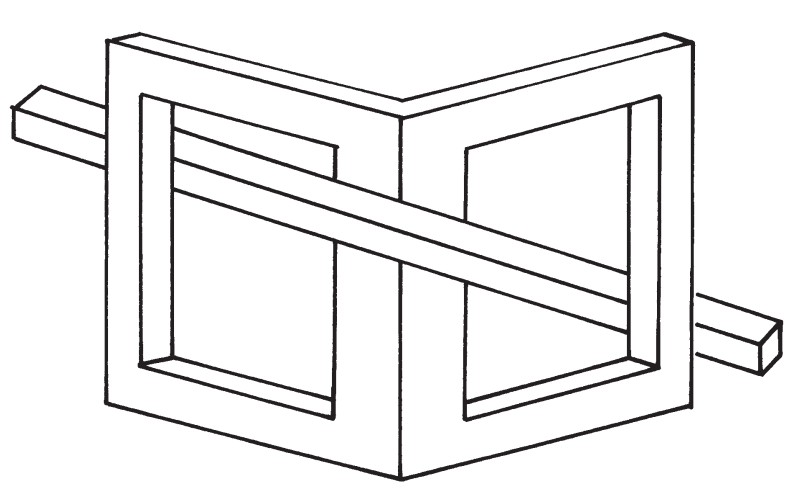

(a)

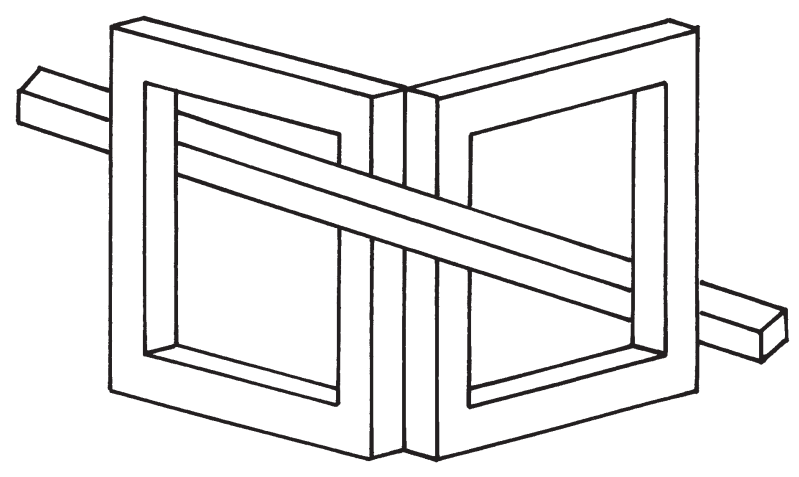

(b)

Fig. 6. Anomalous pictures: (a) unrealizable; (b) realizable.

The additional rod is partly hidden by the frames of the windows. Assumption 1 implies that the rod is not disconnected in the hidden part. Hence, we do not consider the case where the rod consists of several connected components or the case where the rod is curved.

First, consider the pictures in Fig. 5(a) and Fig. 6(a). The object drawn in Fig. 5(a) has exactly four degrees of freedom. In order to reconstruct the object from this picture, we first specify the heights of three vertices of one wall, say the vertices $v_{1}, v_{2}$ and $v_{3}$ on the left wall (in the figure the vertex $v_{i}$ is indicated by $i$ ), and next specify the height of another vertex, say the vertex $v_{4}$. Note that the height of $v_{4}$ should be given in such a way that $v_{4}$ is farther than the plane passing through $v_{1}, v_{2}$ and $v_{3}$, because otherwise the visible part and the rear part would be reversed. Therefore, the vertical edge at the center is necessarily a ridge, which implies that the straight rod cannot be inserted in the way as shown in Fig. 6(a). Hence, we can judge that the object drawn in Fig. 6(a) is not realizable in the three-dimensional space.

Next consider the pictures in Fig. 5(b) and Fig. 6(b). The object drawn in Fig. 5(b) has more than four degrees of freedom. Actually each of the left and right tight components has four degrees of freedom, and they share two common vertices along the vertical edge at the center, and hence the total degrees of freedom is 6 . We can utilize these degrees of freedom so that the straight rod can go through the two windows just in the way as shown in Fig. 6(b). Actually the object can be reconstructed in the following way.

First let us fix the heights of vertices $v_{1}$ and $v_{2}$, and then give the height of $v_{3}$ and $v_{5}$ in such a way that the vertical edge $v_{1} v_{2}$ forms a valley to the viewer, that is, the vertex $v_{5}$ is nearer to the viewer than the plane containing $v_{1}, v_{2}$ and $v_{3}$. There are still two more degrees of freedom; one in the left tight component and one in the right tight component. So finally let us fix the heights of $v_{4}$ and $v_{6}$ so that $v_{4}$ is nearer to the viewer than the plane containing $v_{1}, v_{2}$ and $v_{3}$, and $v_{6}$ is nearer than the plane containing $v_{1}, v_{2}$ and $v_{5}$.

Since the leftmost and rightmost portions are nearer to the viewer than the central portion, a straight rod can go through the two windows just as shown in Fig. 6(b). Thus, the picture in Fig. 6(b) is realizable, and we can actually construct an anomalous solid.

Figure 7 shows an example of an anomalous solid generated in this way. Figure 7(a) is the photograph of the solid seen from the same view point as the picture in Fig. 6(b). If we see this object from the left side, we get the image as shown in Fig. 7(b). In other words, if we see the object in Fig. 7(b) from right, we get the image shown in Fig. 7(a). 


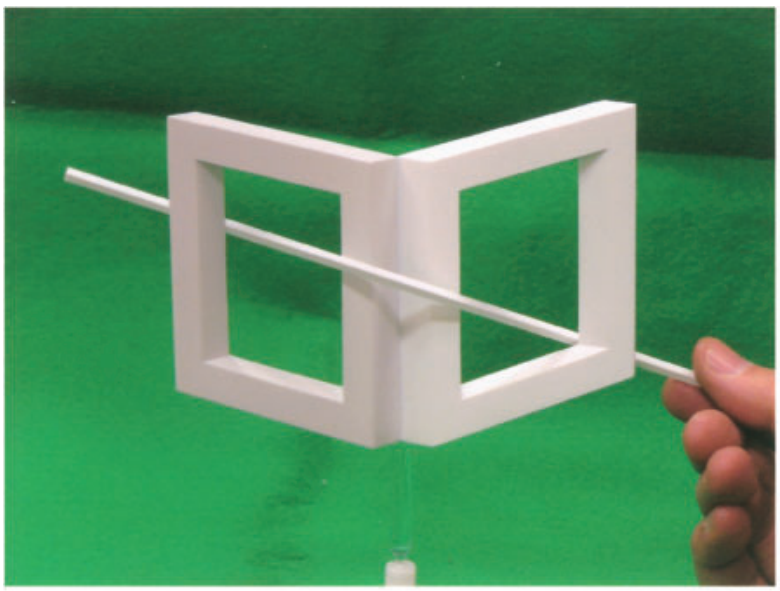

(a)

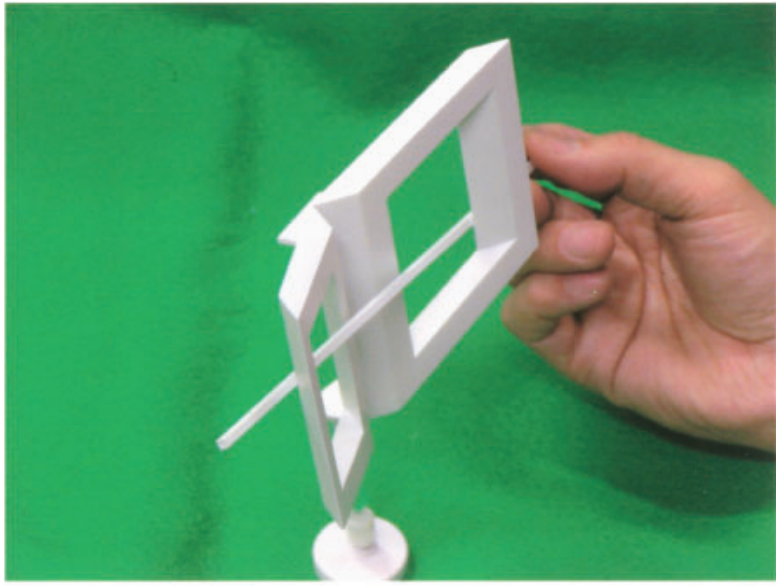

(b)

Fig. 7. Anomalous solid.

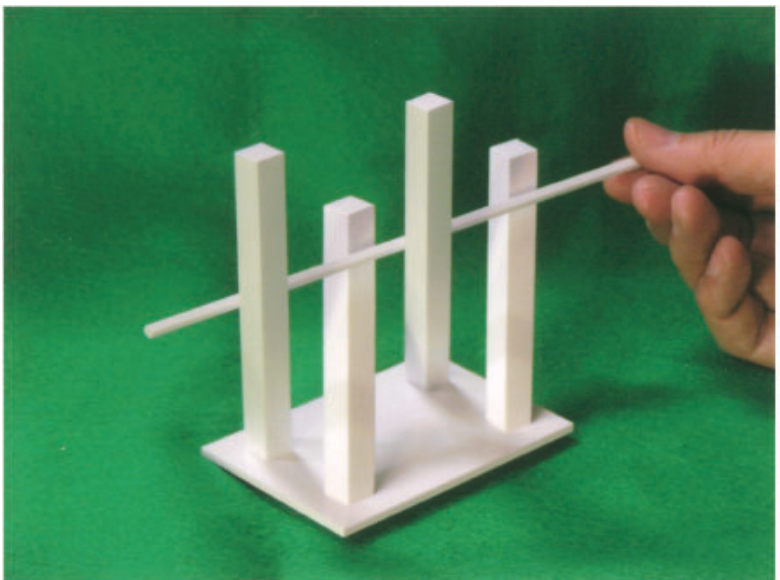

(a)

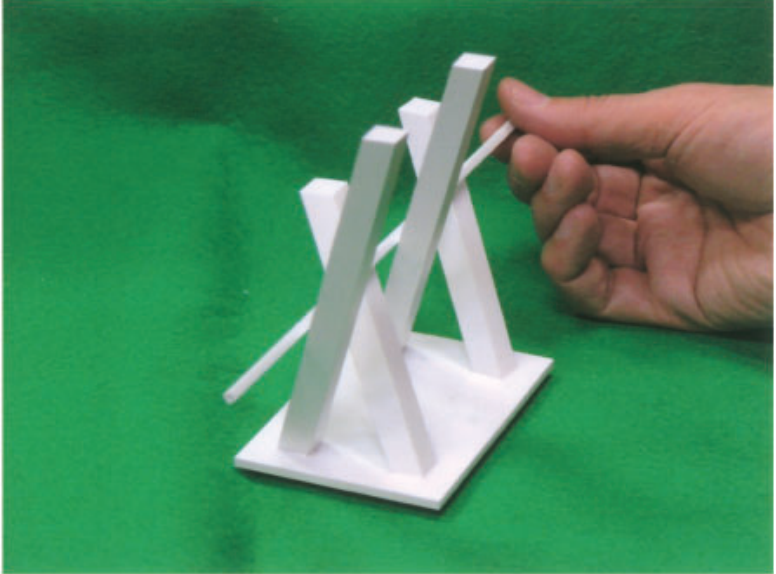

(b)

Fig. 8. Anomalous solid with four columns.

Next consider the object shown in Fig. 4. As we have already seen, this object has eight degrees of freedom. Therefore, we have much more freedom in choosing the actual shape of the object. Indeed, we can choose the object shape in such a way that a straight rod can go through between the four columns as shown in Fig. 8(a); this is an anomalous solid because the rod seems to hide the nearer columns and seems to be hidden by the farther columns.

The true shape of this object is as shown in Fig. 8(b). From this picture, we can see that the four columns are not vertical; two of them bend in one way while the other two bend in the opposite way.

Whether a picture or a solid is anomalous or not much depends on the psychological aspect of human vision, and hence it is impossible to define these concepts in a purely mathematical manner. However, we can state at least the following principle for constructing anomalous solids from anomalous pictures.

Design Principle for Anomalous Solids If a given picture has exactly four degrees of freedom in the choice of object, it is almost impossible to generate an anomalous solid in the three-dimensional space. On the other hand, if a given picture has two or more tight components, it might be possible to generate the associated anomalous solid by choosing the heights of the vertices appropriately.

This principle is vague and far from mathematical. However, this kind of vagueness seems unavoidable for anomalous solids, because whether a solid is normal or anomalous depends on psychological phenomena in human perception. Nevertheless, the author believes that, though the above design principle is vague, it is still useful to discover anomalous solids.

\section{Concluding Remarks}

We considered the degrees of freedom in the choice of three-dimensional polyhedral objects represented by a picture, 
and proposed the decomposition of an object into tight components. On the basis of this consideration we next pointed out that the chance for us to be able to construct an anomalous solid is much greater when the object has two or more tight components than when the object consists of a single tight component.

This characterization of anomalous solids is very weak and far from mathematical. However, this is due to the nature of human perception. Nevertheless, this characterization is useful to design new anomalous solids.

This work is supported by the Grant-in-Aid for Scientific Research of the Japanese Ministry of Education, Culture, Sports, Science and Technology, and by the 21st Century COE Program on Information Science and Technology Strategic Core of the University of Tokyo.

\section{REFERENCES}

[1] Anno, M., Book of ABC (in Japanese). Fukuinkan-Shoten, Tokyo (1974).

[2] Clowes, M. B., "On seeing things," Artificial Intelligence, 2: 99-116 (1971).

[3] Cowan, T. M., "Theory of braids and the analysis of impossible objects," J. Math. Psychol., 11: 190-212 (1974).

[4] Cowan, T. M., "Organizing the properties of impossible figures," Perception, 6: 41-56 (1977).

[5] Draper, S. W., "The Penrose triangle and a family of related figures," Perception, 7: 283-296 (1978).

[6] Draper, S. W., "The use of gradient and dual space in line-drawing interpretation," Artificial Intelligence, 17: 461-508 (1981).

[7] Ernst, B., The Eye Bequiled—Optical Illusions. Benedikt Taschen Verlag, Köln (1992).

[8] Escher, M. C., Graphic. Benedikt Taschen Verlag, Köln (1993).

[9] Gregory, R. L., The Intelligent Eye. Third Edition, Weidenfeld and Nicolson, London (1971).

[10] Huffman, D. A., "Impossible objects as nonsense sentences," B. Meltzer and D. Michie (eds.), Machine Intelligence 6, Edinburgh University Press, Edinburgh, pp. 295-323 (1971).

[11] Penrose, L. S., and Penrose, R., "Impossible objects-A special type of visual illusion," British Journal of Psychology, 49: 3133 (1958).

[12] Robinson, J. O., The Psychology of Visual Illusion. Hutchinson, London (1972).

[13] Sugihara, K., "Classification of impossible objects," Perception, 11: 65-74 (1982).

[14] Sugihara, K., Machine Interpretation of Line Drawings. MIT Press, Massachusetts (1984).

[15] Sugihara, K., "Detection of structural inconsistency in systems of equations with degrees of freedom and its applications," Discrete and Applied Mathematics, 10: 297-312 (1985).

[16] Sugihara, K., "Three-dimensional realization of anomalous pictures-An application of picture interpretation theory to toy design," Pattern Recognition, 30: 1061-1067 (1997).

[17] Sugihara, K., "Mathematics of pictures of impossible objects-Human vision vs robot vision," Sugaku Expositions, 17: 37-49 (2004).

[18] Sugihara, K., and Hayakawa, S., Joy of Impossible Objects (Damashi-E de Asobo). Iwanami, Tokyo (1997).

[19] Térouanne, E., "Impossible figures are interpretation of polyhedral figures," J. Math. Psychol., 27: 370-405 (1983). 